\title{
Novel Adaptive Neuro-Fuzzy based Edge Detection Technique
}

\author{
Suryakant \\ Department of \\ Computer Science \&Engg. \\ N.I.T Jalandhar
}

\author{
Renu Dhir \\ Department of \\ Computer Science \&Engg. \\ N.I.T Jalandhar
}

\begin{abstract}
Digital image processing is a subset of the electronic domain where the image is converted to an array of small integers, called pixels, symbolizing a physical quantity such as scene radiance, stored in a digital memory, and processed by computer or other digital hardware. Interest in digital image processing methods stems from two principals applications areas: improvement of pictorial information for human interpretation; and processing of image data for storage, transmission, and representation for autonomous machine perception. Edges characterize boundaries and edge detection is one of the most difficult tasks in image processing hence it is a problem of fundamental importance in image processing. Edges in images are areas with strong intensity contrasts and a jump in intensity from one pixel to the next can create major variation in the picture quality. Edge detection of an image significantly reduces the amount of data and filters out useless information, while preserving the important structural properties in an image.

This research problem deals with a novel adaptive neuro-fuzzy inference system (ANFIS) for edge detection in digital images. The internal parameters of the proposed ANFIS edge detector are optimized by training using by proposed training pattern. The edges are directly determined by ANFIS network
\end{abstract}

\section{General Terms}

Image, Edge Detection, Fuzzy Logic, Neural Network,Adaptive Neuro-Fuzzy Inference System,Sobel operator, Roberts operator

\section{Keywords}

Edge Detection, Fuzzy Logic, Neural Network, Adaptive Neuro-Fuzzy Inference System, ANFIS

\section{INTRODUCTION}

Edge detection is a often performed operation in image processing applications because it is normally the initial operation that is carried out in advance of other image processing tasks such as image segmentation, boundary detection, object recognition and classification, image registration, etcetera.

To extract the edges from the images, derivative edge detection operators, such as Sobel operator, Roberts operator, and Laplacian operator, are frequently used. However, due to the fact the images of real scenes oftentimes consists of data that is uncompleted and ambiguous [1], the processed results using the methods stated previously are thought to be undesirable. Because the gray level edge is in fact a fuzzy concept, in the last few years, the image edge detection based on the neural networks and fuzzy logic systems have attracted attention of many researchers [2-8]. This is primarily the fact that Neuro-Fuzzy (NF) systems are very well suited tools to deal with precariousnessseen in the process of extracting useful information from images since NF systems unite the capability of neural networks to learn from examples and the capability of fuzzy logic systems to model the precariousness and impreciseness. Therefore, NF systems may be applied as powerful tools for edge detection provided thatmore appropriate network topologies and training strategies are opted.

In the system described in [9], all inputs to the fuzzy inference systems (FIS) system are obtained by applying to the original image a high-pass filter, a first-order edge detector filter (Sobel operator) and a low-pass (mean) filter. The whole structure is then tuned to function as a contrast enhancing filter and, in another problem, to segment images in a specified number of input classes. The adopted fuzzy rules and the fuzzy membership functions are specified according to the kind of filtering to be executed.

In the system described in [7], present a novel adaptive neurofuzzy inference system (ANFIS) for edge detection in digital images. The internal parameters of the proposed ANFIS edge detector are optimized by training using very simple artificial images.

In [8] a new edge detection technique is proposed basis on the BP neural network. Here, it is classified the edge patterns of binary images into 16 possible types of visual patterns. In the following, after training the pre-defined edge patterns, the BP neural network is applied to correspond any type of edges with its related visual pattern

In $[5,6]$ novel method based on fuzzy logic reasoning strategy is proposed for edge detection in digital images without determining the threshold value. The proposed approach begins by segmenting the images into regions using floating $3 \times 3$ binary matrix. The edge pixels are mapped to a range of values distinct from each other

In this paper, we present a novel adaptive neuro-fuzzy Inference system (ANFIS) for edge detection of an image. The key features of our approach which differentiate us from others is the use of image content and adaptive neuro-fuzzy Inference system for edge detection of application-specific image. The result has been compared with the standard algorithms. The proposed technique can be extended for color images as well.

In this paper we proposed a novel adaptive Neuro-Fuzzy Inference System (ANFIS) for edge detection in digital images. In the proposed method, the edges in the image are directly determined by an ANFIS network. The proposed ANFIS edge detector is tested on various images having different image properties and also compared with popular edge detectors (i.e. Sobel \& Roberts). Experimental results show that the proposed ANFIS edge detector exhibits much 
better performance than the conventional (i.e. Sobel \& Roberts) operators and may efficiently be used for the detection of edges in digital images.

\section{ANFIS METHOD}

Adaptive neuro fuzzy inference system (ANFIS) combines the advantage of fuzzy logic and neural networks. The ANFIS is a fuzzy rule- based network having neural network's learning ability. The major characteristic of the network is that no preassigning and designing of the rules are needed. The rules are constructed automatically during the online process. Two learning phases, the structure identification along with the parameter learning phases are espoused online for the construction task. The structure identification ascertain the adequate number of rules needed i.e., finding how many rules are necessary and be adequate to properly model the available data and the number of membership functions for input and output variables. Fig. 1 displays the diagram of the proposed ANFIS. The inputs and their membership functions appear to the left of the ANFIS structural characteristics, while outputs and their membership functions appear on the right.

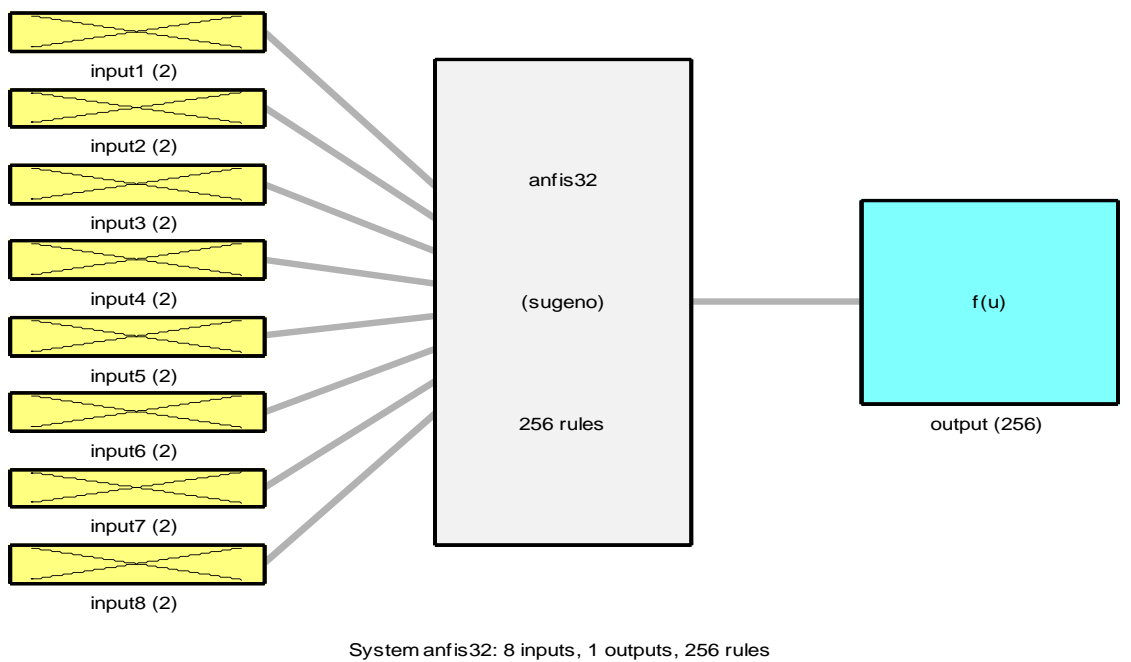

Fig 1:Diagram of the proposed ANFIS

\subsection{Proposed method}

The proposed ANFIS detector is a first-order Sugeno type fuzzy inference system with 8-inputs and 1-output. Each input has 2 triangular type membership functions and the output has a constant membership function. Therefore 256 rules were taken. The no of output membership functions are 256 and there is only one output. This is because ANFIS does not allow any rule sharing. There are two options available for designing a FIS in the ANFIS editor. They are [A] Grid partition method and [B] subtractive clustering method. In this proposed work grid partition method is used. Hybrid algorithm is used for training the model. The proposed algorithm uses ANFIS GUI of MATLAB for parameter adjustment. Figure-2 represents the ANFIS structure.

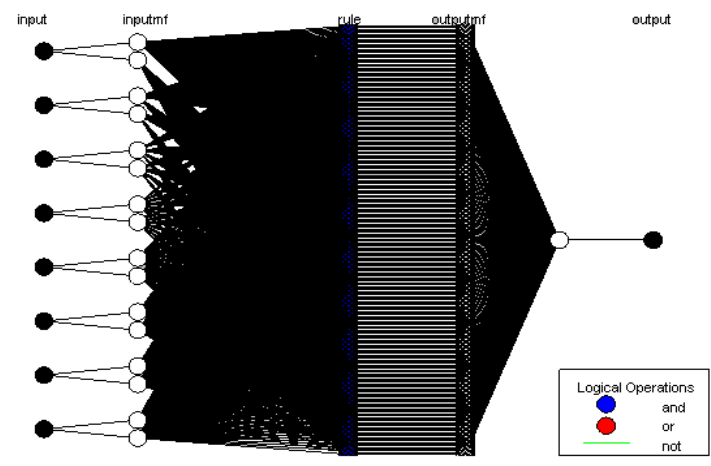

Figure 2: ANFIS Structure
To detect edges in a gray level image, we firstly binarize the image, and then the binary image disintegrate to $3 \times 3$ windows and generates a set of image pattern. The edge patterns in binary images are classified into 32 categories, as shown in figure 3, and then train the ANFIS on these patterns. The blank elements in each $3 \times 3$ window indicate white (pixels value: 1s) in binary images, whereas the dark elements indicate black (pixels value: $0 \mathrm{~s}$ ).

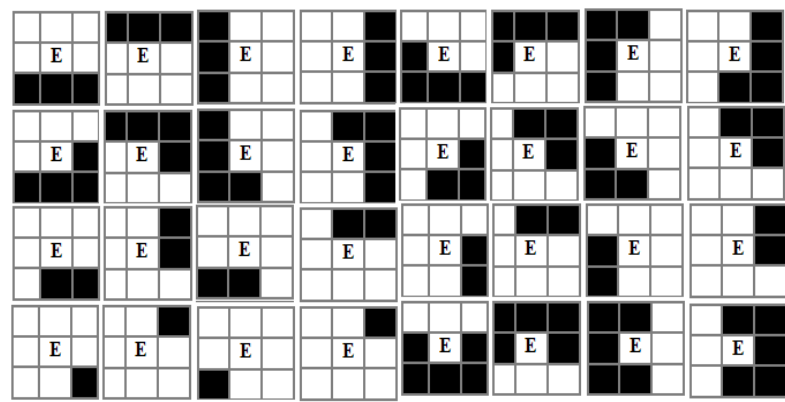

Figure 3:Possible type of input patterns

\subsection{Designed ANFIS}

The most difficult part of any ANFIS training problem is defining the proper training set. A simple method is recommended for the edge detection training problem. Training of ANFIS can be accomplished by preparing a dataset in the following manner. 
Table 1. Proposed training pattern

\begin{tabular}{|c|c|}
\hline Input pattern & Output pattern \\
\hline $\begin{array}{llllllll}1 & 1 & 1 & 1 & 1 & 0 & 0 & 0\end{array}$ & 1 \\
\hline $\begin{array}{llllllll}0 & 0 & 0 & 1 & 1 & 1 & 1 & 1\end{array}$ & 1 \\
\hline $\begin{array}{llllllll}0 & 1 & 1 & 0 & 1 & 0 & 1 & 1\end{array}$ & 1 \\
\hline 1101101110 & 1 \\
\hline $\begin{array}{llllllll}0 & 0 & 1 & 0 & 1 & 0 & 1 & 1\end{array}$ & 1 \\
\hline 11001010100 & 1 \\
\hline $\begin{array}{llllllll}0 & 1 & 1 & 0 & 1 & 0 & 0 & 1\end{array}$ & 1 \\
\hline 1000100110 & 1 \\
\hline $\begin{array}{lllllllll}0 & 0 & 0 & 0 & 1 & 1 & 1 & 1\end{array}$ & 1 \\
\hline $\begin{array}{llllllll}1 & 1 & 1 & 0 & 1 & 0 & 0 & 0\end{array}$ & 1 \\
\hline $\begin{array}{llllllll}0 & 0 & 0 & 1 & 0 & 1 & 1 & 1\end{array}$ & 1 \\
\hline $\begin{array}{llllllll}1 & 1 & 1 & 1 & 0 & 0 & 0 & 0\end{array}$ & 1 \\
\hline $\begin{array}{llllllll}1 & 0 & 0 & 1 & 0 & 1 & 1 & 1\end{array}$ & 1 \\
\hline 1111101100 & 1 \\
\hline $\begin{array}{llllllll}0 & 0 & 1 & 0 & 1 & 1 & 1 & 1\end{array}$ & 1 \\
\hline $\begin{array}{llllllll}1 & 1 & 1 & 0 & 1 & 0 & 0 & 1\end{array}$ & 1 \\
\hline $\begin{array}{llllllll}0 & 1 & 1 & 0 & 1 & 1 & 1 & 1\end{array}$ & 1 \\
\hline $\begin{array}{llllllll}1 & 1 & 1 & 0 & 1 & 0 & 1 & 1\end{array}$ & 1 \\
\hline $\begin{array}{llllllll}1 & 1 & 1 & 1 & 1 & 0 & 0 & 1\end{array}$ & 1 \\
\hline $\begin{array}{llllllll}0 & 0 & 1 & 1 & 1 & 1 & 1 & 1\end{array}$ & 1 \\
\hline $\begin{array}{llllllll}1 & 1 & 1 & 1 & 1 & 0 & 0\end{array}$ & 1 \\
\hline 111110110 & 1 \\
\hline $\begin{array}{llllllll}1 & 1 & 0 & 1 & 0 & 1 & 1 & 1\end{array}$ & 1 \\
\hline $\begin{array}{llllllll}1 & 0 & 0 & 1 & 1 & 1 & 1 & 1\end{array}$ & 1 \\
\hline $\begin{array}{llllllll}0 & 0 & 0 & 0 & 0 & 1 & 1 & 1\end{array}$ & 1 \\
\hline $\begin{array}{llllllll}0 & 0 & 1 & 0 & 1 & 0 & 0 & 1\end{array}$ & 1 \\
\hline 1111000000 & 1 \\
\hline 10010100 & 1 \\
\hline $\begin{array}{llllllll}0 & 1 & 1 & 1 & 1 & 1 & 1 & 1\end{array}$ & 1 \\
\hline $\begin{array}{llllllll}1 & 1 & 1 & 1 & 1 & 0 & 1 & 1\end{array}$ & 1 \\
\hline $\begin{array}{lllllll}1 & 1 & 1 & 1 & 1 & 1 & 0\end{array}$ & 1 \\
\hline $\begin{array}{llllllll}1 & 1 & 0 & 1 & 1 & 1 & 1 & 1\end{array}$ & 1 \\
\hline
\end{tabular}

The designed ANFIS system is given eight inputs and generates one output. The Eight inputs are the eight pixel values $(\mathrm{p} 1, \mathrm{p} 2, \mathrm{p} 3, \mathrm{p} 4, \mathrm{p} 6, \mathrm{p} 7, \mathrm{p} 8, \mathrm{p} 9)$ of the window mask used. Take an image object to be learned and slide it from point to point across all locations of a window which will be the input window to the pattern detection network. We also notice that the two margin rows and two margin columns around the image cannotbe processed and a kind of padding required. When the whole binary image is scanned by the input pixel window, we obtain the edges.

\begin{tabular}{|c|c|c|}
\hline P1 & P2 & P2 \\
\hline P4 & P5 & P6 \\
\hline P7 & P6 & P9 \\
\hline
\end{tabular}

Fig 4: Floating 3x3 pixel window mask

\section{RESULTS}

The proposed fuzzy edge detection method was simulated using MATLAB on different images, its performance are compared to that of the Sobel and Roberts operators. In first example, Diagonal-lines image is processed. The main image is shown in Figure 5(a). The edges extracted by the proposed algorithm are shown in Figure 5(b) Finally, The extracted edges by the Robert and Sobel methods are shown in Figure 5(c) and 5(d).As the Figure shown, the proposed algorithm detects much more edge pixels, and superiority of the proposed algorithm is clear.
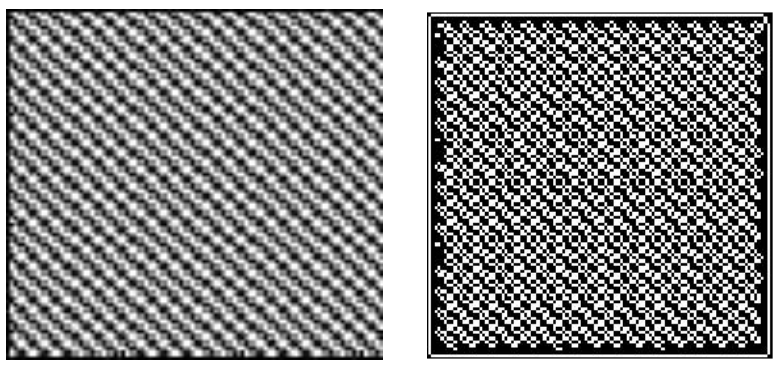

Fig 5: (a) Diagonal-lines image Fig 5 :(b) proposed ANFIS method Results
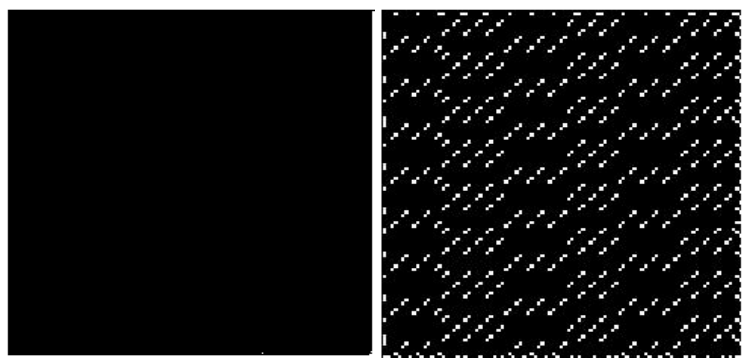

Fig 5: (c) Roberts edge detector Fig 5: (d) Sobel edge detector

Similarly, samples for a set of six test images are shown in Figure 6 (a). The sample output of the proposed ANFIS technique is shown in Figure 6 (b). The edge detection based on Roberts and Sobel operators using the image processing toolbox in MATLAB with threshold automatically estimated from image's binary value is illustrated in figure 6 (c) and 6 (d). The resulting images generated by the ANFIS method seem to be much smoother with less noise (no spurious edges) and has an exhaustive set of fuzzy conditions which helps to provide an efficient edge representation for images with a very high efficiency than the conventional gradient-based methods (Sobel and Roberts methods). 


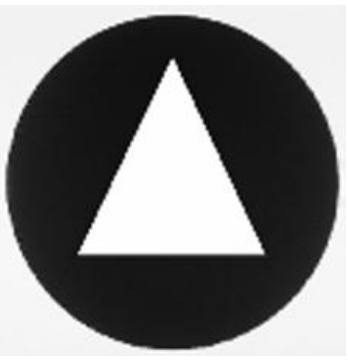

(a)

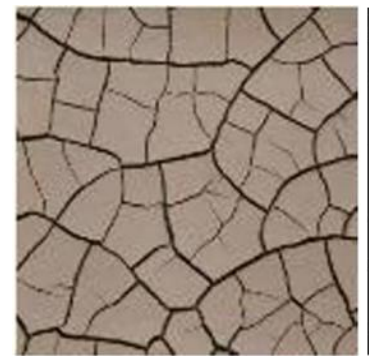

(a)

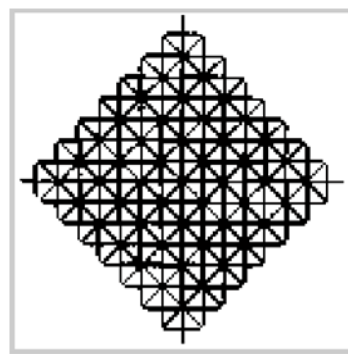

(a)

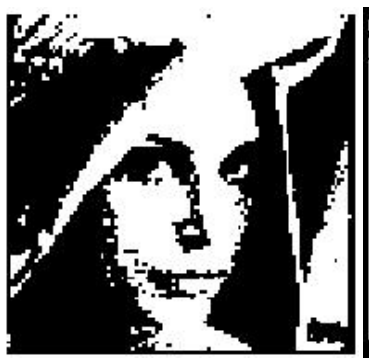

(a)

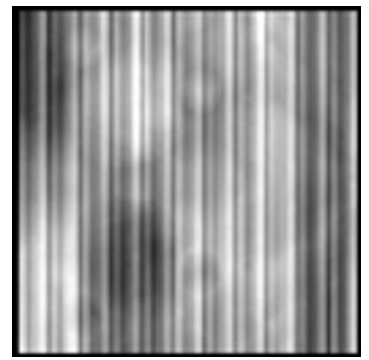

(a)

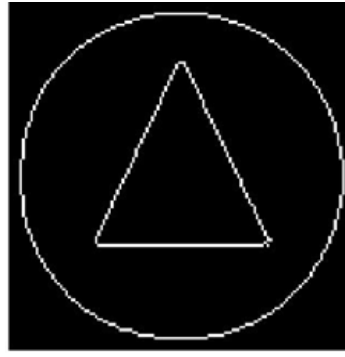

(b)

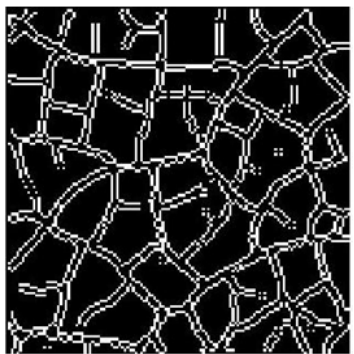

(b)

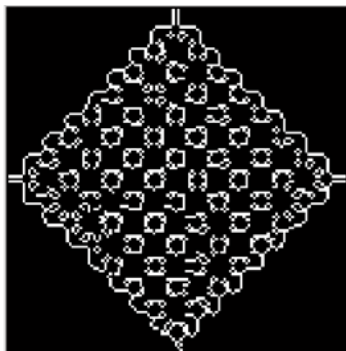

(b)

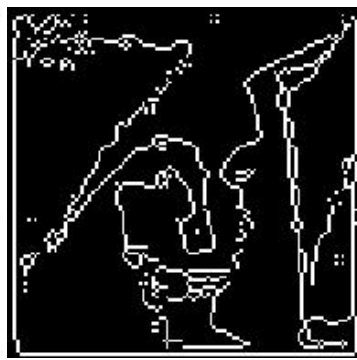

(b)

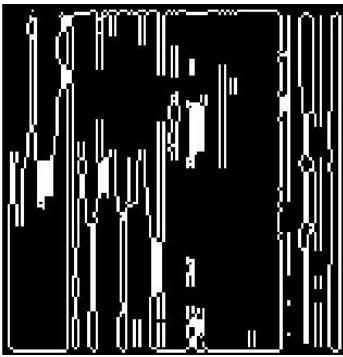

(b)

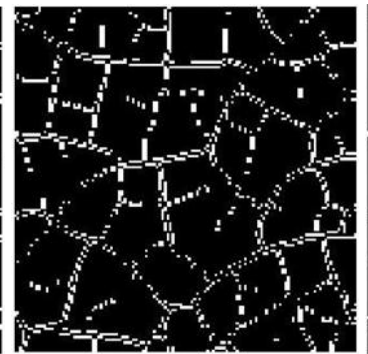

(c)

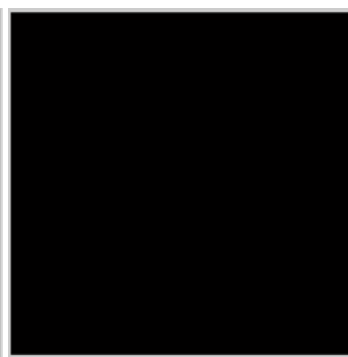

(c)

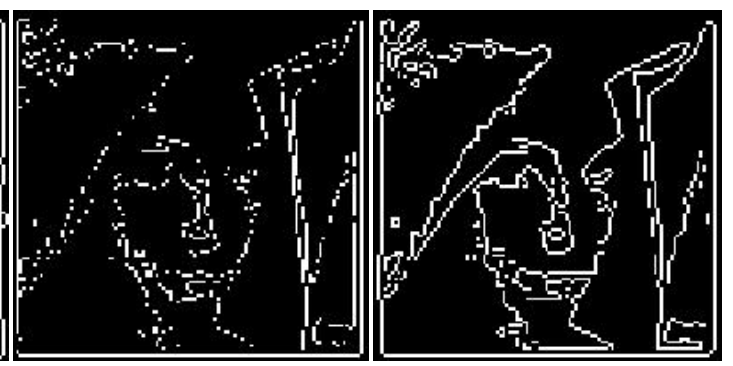

(d)

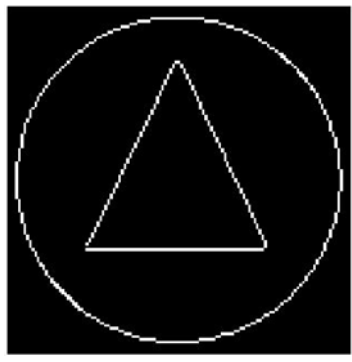

(d)

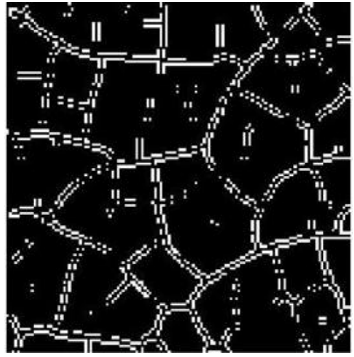

(d)

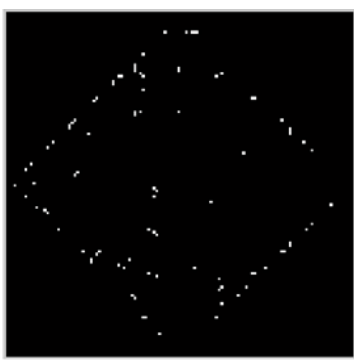

(c)

(c)

(d)

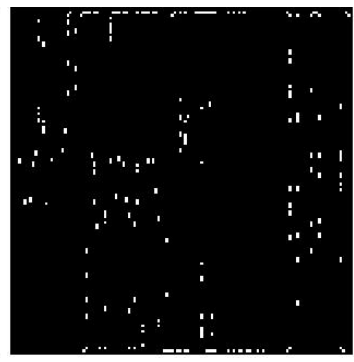

(d) 


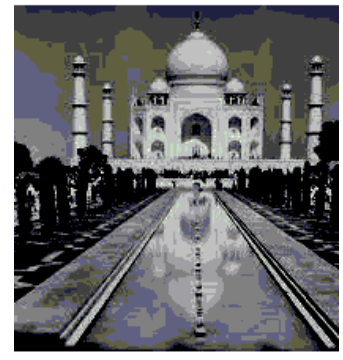

(a)

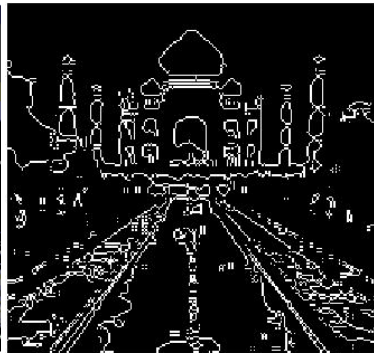

(b)

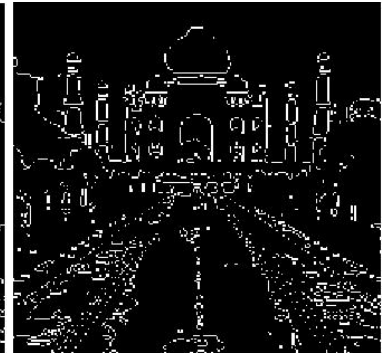

(c)

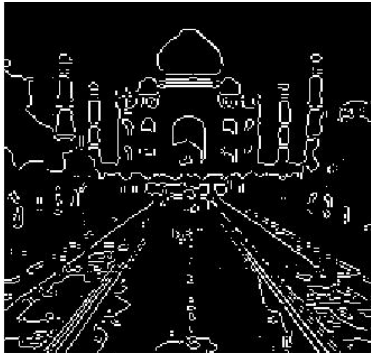

(d)

Figure 6: (a) Original image (b) Proposed ANFIS method Results (c) Roberts edge detector (d) Sobel edge detector

\section{CONCLUSION}

Edge detection is a problem of fundamental importance in image analysis. In typical images, edges characterize object boundaries and are therefore useful for segmentation, registration, and identification of objects in a scene. Edge detection of an image reduces significantly the amount of data and filters out information that may be regarded as less relevant, preserving the important structural properties of an image Fuzzy set theory plays an important role in dealing with uncertainty when making decisions in medical applications. Fuzzy logic enables us to use the uncertainty in the classifier design and consequently increase the credibility of the system output. The proposed technique involves training the ANFIS edge detector for Edge detection.

ANFIS is the fuzzy logic based paradigm that grasps the learning abilities of ANN to enhance the intelligent system's performance using a priori Knowledge. Using a given input/output data set, ANFIS constructs a fuzzy inference system (FIS) whose membership function parameters are tuned (adjusted) using either a backpropagation algorithm alone, or in combination with a least squares type of method. This allows the fuzzy systems to learn from the modeled data. The parameters associated with the membership functions will change through the learning process. The computation of these parameters (or their adjustment) is facilitated by a gradient vector, which provides a measure of how well the fuzzy inference system is modeling the input/output data for a given set of parameters. Once the gradient vector is obtained, any of several optimization routines could be applied to adjust parameters that will reduce some error measure (usually defined by the sum of the squared differences between actual and desired response).

These techniques provide a method for the fuzzy modeling procedure to learn information about a data set, in order to compute the membership function parameters that best allow the associated fuzzy inference system to track the given input/output data. This learning method works similarly to that of neural network.

A novel ANFIS edge detector for digital images presented. The advantages of the proposed ANFIS edge detector may be summarized as follows:

a) It has a very simple structure. Its fundamental building block is a 8-input 1-output ANFIS system. This greatly simplifies implementation.

b) It is trained by using proposed training set.

It is concluded that the novel ANFIS edge detector can be used for efficient extraction of edges in digital images. This technique uses the edge strength information derived using strong Fuzzy If then rule to avoid detection of spurious edges corresponding to noise, which is often the case with conventional gradient-based techniques (Sobel and Roberts).
Through the simulation results, it is shown that ANFIS Edge detector's application on digital image improves the quality of edges as much as possible compared to the Sobel and Roberts methods. This algorithm is suitable for applications in various areas of digital image processing such as face recognition, fingerprint identification, remote sensing and medical imaging where boundaries of specific regions need to be determined for further image analysis.

\section{FUTURE SCOPE}

The proposed technique to find more fine edges using ANFIS edge detector is restricted only to gray scale image. This can be further extended to color image, where edge detection task may become significantly more complex .Moreover, modification of training set so as to reduce inclusion in the output image of pixels not belonging to edges which can produce better result.

\section{REFERENCES}

[1] Berzins, 1984 "V.Accuracy of Laplacian Edge Detector Computer Vision, Graphics, and Image Processing,",

[2] D.H. Ballard and C.M. Brown 1982.," Computer Vision," Prentice--Hall, New Jersey,

[3] J. M. S. Prewitt 1970, "Picture Processing and Psychpictorics," B.S. Lipkin and A. Rosenfeld Eds, Academic Press, New York,.

[4] R. O. Duda. and P. E. Hart 1973, "Pattern Classification and Scene Analysis," Wiley, New York,

[5] Abdallah A. Alshennawy, And Ayman A. Aly 2009, "Edge Detection In Digital Images Using Fuzzy Logic Technique" . World Academy of Science Engineering and Technology

[6] Begol, Moslem and Maghooli,Keivan 2011, "Improving Digital Image Edge Detection by Fuzzy Systems World Academy of Science, Engineering and Technology.

[7] Lei Zhang, Mei Xiao, Jian Ma and Hongxun Song 2009 " Edge Detection by Adaptive Neuro-Fuzzy Inference System"

[8] Hamed Mehrara, Mohammad Zahedinejadand Ali Pourmohammad 2009, "Novel Edge Detection Using BP Neural Network Based on Threshold Binarization" Second International Conference on Computer and Electrical Engineering.

[9] Tizhoosh H.R 2002, "Fast fuzzy edge detection", Proceedings of Fuzzy Information Processing Society, 